Studies on regulated expression of plant defense genes

\title{
Kazuyuki HIRATSUKA*
}

hiratsuka-kazuyuki-pz@ynu.ac.jp

*Graduate School of Environment and Information Sciences, Yokohama National University, Yokohama 240-8501, Japan

\section{Introduction}

To ward off pathogen infection, plants rely on various disease responses at the cell/tissue level as part of their immune system and have long been attractive topics for research. Extensive research has been conducted on the interaction between host plants and pathogens and on plant recognition of pathogen infection and the signal transduction system as plant molecular biology has advanced (Ohashi 1996). Later, genes involved in disease responses in plants were discovered and studied using molecular biological methods from the 1980s, and since the 1990s, salicylic acid and jasmonic acid have been recognized as plant hormones related to the control of defense responses. Work on these molecules has contributed to the elucidation of regulatory mechanisms involved in the expression of defense-response genes in higher plants. Research on the regulation of gene expression in higher plants has progressed dramatically, and cis-regulatory elements and cognate sequence-specific DNA-binding proteins have been identified as trans-regulators through the analysis of the regulatory mechanisms of gene promoters. Research on the practical application of disease-responsive gene promoters and the evaluation and search for resistance inducers to use in the field has also flourished. In this paper, I will introduce related studies in which I have been involved, mainly on transcription factors and applied research using the bioluminescence reporter method. 
2. Transcriptional regulators involved in defense response gene expression

Research on gene expression regulation in the 1980s progressed from functional analysis of expression control regions to identification of cis-regulatory elements within the promoter sequence. Subsequently, research was conducted on sequence-specific DNA-binding proteins as trans-regulators that bind to cis-regulatory elements. Many DNA-binding proteins as trans-regulators were isolated and identified as transcription-factors using a search method that uses binding activity to specific DNA sequences and a method based on DNA sequence homology (Hiratsuka and Chua 1997; Ramachandran et al. 1994).

GT-1, which was first identified as a type of plant-specific transcription factor trihelix protein, was initially identified from a nuclear extract of tobacco as a DNA-binding protein that binds to a light-inducible gene promoter. A tobacco cDNA was isolated with a method using the target sequence as a probe (Gilmartin et al. 1992). The identification of a homologous gene in Arabidopsis thaliana led to the further characterization of GT-1, including detailed binding characteristics to cis-regulatory factors, nuclear translocation ability, and regulation of binding activity by phosphorylation (Hiratsuka et al. 1994; Maréchal et al. 1999). Subsequent studies showed that it also functions as a binding factor for the tobacco $P R$-1a promoter, which is a typical disease-responsive gene promoter, and showed a relationship between light-responsive gene control and defense-responsive gene expression at the transcriptional level (Buchel et al. 1999). Extensive research was also carried out on linking cis-regulators to signal transduction systems, and new findings on signal transduction systems and cis-elements mediated by calcium and cyclic GMP were discovered (Neuhaus et al. 1997; Wu et al. 1996).

A large-scale analysis of genes highly expressed in germ cells of the trumpet lily (Lilium longiflorum) was carried out, focusing on the genes, which show remarkable antimicrobial activity in its floral organs (Morohashi 
et al. 2000). One of the genes highly expressed in reproductive organs was discovered to encode LISCL (Lilium longiflorum Scarecrow-like), one of the novel GRAS proteins, and investigated in detail. The GRAS proteins are a group of regulator of higher plants and their mechanism of action was unknown. The LlSCL was shown to be localized in the nucleus and function as a transcriptional activator (Morohashi et al. 2003). Subsequent studies showed that the LISCL homologous gene in Arabidopsis is involved in the defense response to saprophytic pathogens such as Botrytis cinerea, and the LlSCL group of genes are regarded as transcriptional regulators involved in defense responses specific to higher plants (Fode et al. 2008).

3. Firefly luciferase assay as a tool to study regulation of defense-response gene expression

We worked on the technological development of a bioluminescence reporter using firefly luciferase that enables continuous monitoring of gene expression control in plants (Hiratsuka 1997; Millar et al. 1992). The luciferase reporter assay was originally used in studies targeting light-inducible gene promoters. For developing luciferase to study defense gene expression in higher plants, first we exploited the tobacco $P R$-1a gene promoter using a promoter sequence obtained from the genomic DNA of tobacco BY-2 cells. We made a fusion gene between the firefly luciferase gene and the $P R$-1a promoter sequence of about 1600 base pairs, introduced it into BY-2 cells and tobacco plants to assess the activity of the $P R$-1a promoter as a change in luminescence activity. The results showed that salicylic acid treatment significantly increased the bioluminescence intensity and that samples could be continuously observed nondestructively (Hiratsuka 1997, Watakabe et al. 2011). We also constructed a fusion gene between the firefly luciferase gene and the promoter region of Arabidopsis thaliana MPK3 gene, which encodes a protein kinase involved in signal transduction of defense responses. Using these transgenic Arabidopsis plants harboring the bioluminescence reporter gene, we could analyze expression profiles of the 
MPK3 promoter in response to infection by B. cinerea (Tanaka et al. 2006).

We also developed a transient expression assay system using a microprojectile bombardment method. Using the Renilla luciferase gene as an internal standard, we established a dual luciferase assay system for the accurate quantitative evaluation of gene expression. The experimental method is now widely used for transient assay in plant cells (Matsuo et al. 2001). The transient assay system was used to show transcriptional activation of $P R$ - 1 a after salicylate treatment or simultaneous introduction of NPR-1 gene (Ono et al. 2004).

In addition, studies on multicolor luciferase to develop a highly accurate bioluminescence reporter gene system in plants. The two types of bioluminescent genes derived from the click beetle encoding green-luminescent and red-luminescent luciferases, respectively, use luciferin as a luminescent substrate, the same substrate for firefly luciferase. Therefore, the expression levels of two independent genes can be monitored simultaneously by measuring green emission and red emission independently using a spectral filter, which is called a dual color luciferase assay. We were the first to develop this assay system for use in plant tissues, and showed that it is effective for finding and analyzing defense gene inducers (Kusama et al. 2009; Ogura et al. 2005, 2011).

In parallel, we worked on the development of a high-throughput system to screen and evaluate defense gene inducers (plant activator). Arabidopsis seeds harboring a bioluminescence reporter are dispensed into a multi-well plate, then treated with chemicals to be tested. The system allows us to continuously observe many samples nondestructively and is an ideal method for screening chemical libraries or the like for novel compounds that can control plant pathogens. In particular, the fusion gene of the tobacco $P R$ - $1 a$ promoter and firefly luciferase showed better responsiveness to induction stimulation than the endogenous gene of Arabidopsis, and was shown to be suitable for search and evaluation system for defense gene inducers (Ono et al. 2011; Watakabe et al. 2001). In addition, we prepared a fusion gene 
between firefly luciferase and the promoter region of the Vegetative storage protein 1 gene, a responsive gene of jasmonic acid system of Arabidopsis. Using Arabidopsis seedlings harboring the fusion gene and multi-well plates, we demonstrated that the system can be applicable to a high-throughput system for monitoring jasmonate-regulated defense response genes (Kusama et al. 2012).

These systems are also used to evaluate the physiological activity of existing chemicals and the like. We used the system to detect defense gene induction activity from yeast cell wall extracts and suggested that the disease control activity resulted from treatment with yeast cell wall extracts may be due to induction of the defense gene expression (Minami et al. 2011). We also found that tolprocarb, a fungicide for rice blast that acts as a melanin synthesis inhibitor, clearly has resistance-inducing activity and it is also effective in controlling various bacterial diseases (Hagiwara et al. 2020).

The screening system functions effectively as a high-throughput screening system and is utilized for discovering new compounds that activate defense genes of higher plants. In particular, a group of methyl jasmonate agonists discovered using the high-throughput system has no structural similarity to jasmonates and showed a novel type of physiological activity (Tomita et al. 2019).

4. Social implementation of research results

We established Yokohama Biotechnology Co., Ltd., a venture company originating from Yokohama National University, for the purpose of disclosing and utilizing a series of research results on regulating defense-response gene expression and as a contract service for evaluating substances for expression induction of plant-defense-response genes (http://ybt.co.jp/). In particular, the assay system using a bioluminescence reporter has been well-received because it can quantitatively evaluate inducing activity of the substances. So far, contract research for the development of plant defense inducers has been completed for more than 10 domestic companies. 
Acknowledgments: A series of studies was conducted at Rockefeller University, Nara Institute of Science and Technology, and Yokohama National University, with particular guidance and instruction from Drs. Nam-Hai Chua and Yasuo Hotta. At the Faculty of Agriculture, The University of Tokyo, Drs. Yoji Doi, Shuichi Yamashita, Shigetou Namba and Tsuneo Tsuchizaki guided my development as a researcher. The Ministry of Education, Culture, Sports, Science and Technology's Grant-in-Aid for Scientific Research (Dr. Ichiro Uyeda) and the Ministry of Economy, Trade and Industry and the NEDO project (Dr. Takeshi Matsumura) were particularly supportive of the research described. I also express my sincere gratitude for the great encouragement, support and cooperation from many other people that cannot be listed here.

\section{Compliance with ethical standards}

Conflicts of interest The author has no conflicts of interest to declare.

Ethical approval This article does not contain any studies with human participants or animals performed by any of the authors.

\section{References}

Buchel, AS, Brederode FT, Bol JF, Linthorst HJM (1999) Mutation of GT-1 binding sites in the Pr-1A promoter influences the level of inducible gene expression in vivo. Plant Mol Biol 40: 387-396

Fode B, Siemsen T, Thurow C, Weigel R, Gatz C (2008) The Arabidopsis GRAS protein SCL14 interacts with class II TGA transcription factors and is essential for the activation of stress-inducible promoters. Plant Cell 20: 3122-3135

Gilmartin PM, Memelink J, Hiratsuka K, Kay SA, Chua N-H (1992) 
Characterization of a gene encoding a DNA binding protein with specificity for a light-responsive element. Plant Cell 4: 839-849

Hagiwara H, Ogura R, Fukumoto T, Ohara T, Tsuda M, Hiratsuka K (2020) Novel bacterial control agent tolprocarb enhances systemic acquired resistance in Arabidopsis and rice as a second mode of action. J Gen Plant Pathol 86: 39-47

Hiratsuka K (1997) How to observe LUC activity at the cellular level. (in Japanese) Cell engineering separate volume. Plant cell engineering series $6: 80-84$

Hiratsuka K, Chua N-H (1997) Light regulated transcription in higher plants. J Plant Res 110: 131-139

Hiratsuka K, Wu X, Fukuzawa H, Chua N-H (1994) Molecular dissection of GT-1 from Arabidopsis. Plant Cell 6: 1805-1813

Kusama M, Ogura R, Hiratsuka K (2009) Screening and evaluation of plant activators by the bioluminescence reporter system. (in Japanese) $\mathrm{J}$ Pesticide Sci 34: 346-349

Kusama M, Urata N, Ogura R, Ogata S, Hiratsuka K (2012) Development of a promoter-luciferase-based high-throughput system to monitor jasmonate-mediated defense gene expression. Plant Biotechnol 29: $515-520$

Maréchal E, Hiratsuka K, Delgado J, Nairn A, Qin J, Chait BT, Chua N-H (1999) Modulation of GT-1 DNA-binding activity by calcium-dependent phosphorylation. Plant Mol Biol 40: 373-386

Matsuo N, Minami M, Maeda T, Hiratsuka K (2001) Dual luciferase assay for monitoring gene expression in higher plants. Plant Biotechnol 18: 71-75

Millar AJ, Short SR, Hiratsuka K, Chua N-H, Kay SA (1992) Firefly luciferase as a reporter of regulated gene expression in higher plants. Plant Mol Biol Rep 10: 324-337

Minami T, Tanaka T, Takasaki S, Kawamura K, Hiratsuka K (2011) In vivo bioluminescence monitoring of defense gene expression in response to 
treatment with yeast cell wall extract. Plant Biotechnol 28: 481-484

Morohashi K, Bayaarma GV, Takase H, Hotta Y, Hiratsuka K (2000) Large-scale sequencing of meiosis-associated genes from a cDNA library of lily microsporocytes. Plant Biotechnol 17: 131-135

Morohashi K, Minami M, Takase H, Hotta Y, Hiratsuka K (2003) Isolation and characterization of a novel GRAS gene that regulates meiosis-associated gene expression. J Biol Chem 278: 20865-20873

Neuhaus G, Bowler C, Hiratsuka K, Yamagata H, Chua N-H (1997) Phytochrome-regulated repression of gene expression requires calcium and cGMP. EMBO J 16: 2554-2564

Ogura R, Matsuo N, Wako N, Tanaka T, Ono S, Hiratsuka K (2005) Multi-color luciferases as reporters for monitoring transient gene expression in higher plants. Plant Biotechnol 22: 151-155

Ogura R, Matsuo N, Hiratsuka K (2011) Bioluminescence spectra of click beetle luciferases in higher plant cells. Plant Biotechnol 28: 423-426

Ohashi Y (1996) Molecular biology of resistance to pathogen-infection in plants. (in Japanese) Ann Phytopathol Soc Jpn 62: 213-215

Ono S, Tanaka T, Watakabe Y, Hiratsuka K (2004) Transient assay system for the analysis of $P R-1 a$ gene promoter in tobacco BY-2 cells. Biosci Biotechnol Biochem 68: 803-807

Ono S, Kusama M, Ogura R, Hiratsuka K (2011) Evaluation of the use of the tobacco $P R-1$ a promoter to monitor defense gene expression by the luciferase bioluminescence reporter system. Biosci Biotechnol Biochem 75: $1796-1800$

Ramachandran S, Hiratsuka K, Chua N-H (1994) Transcription factors in plant growth and development. Curr Opin Genet Dev 4: 642-646

Tanaka T, Ono S, Watakabe Y, Hiratsuka K (2006) Bioluminescence reporter assay system to monitor Arabidopsis MPK3 gene expression in response to infection by Botrytis cinerea. J Gen Plant Pathol 72: 1-5

Tomita M, Negishi H, Ogura R, Hiratsuka K (2019) Characterization of a novel compound that enhance defense gene expression. (Abstract in 
Japanese) Jpn J Phytopathol 85: 308-309

Watakabe Y, Ono S, Hiratsuka K (2001) Characterization of agents that induce acquired resistance by transgenic plants. (in Japanese) $\mathrm{J}$ Pesticide Sci 26: 296-299

Watakabe Y, Ono S, Tanaka T, Hiratsuka K (2011) Non-destructive bioluminescence detection system for monitoring defense gene expression in tobacco BY-2 cells. Plant Biotechnol 28: 295-301

Wu Y, Hiratsuka K, Neuhaus G, Chua N-H (1996) Calcium and cGMP target distinct phytochrome-responsive elements. Plant J 10: 1149-1154 